\title{
Dosimetric Comparison of Amorphous Silicon EPID and 2D Array Detector for Pre-Treatment Verification of Intensity Modulated Radiation Therapy
}

\author{
Ayman G. Ibrahim¹, Ismail E. Mohamed', Hamdy M. Zidan³ \\ ${ }^{1}$ Radiation Oncology \& Nuclear Medicine Department, Mansoura University Hospitals, Mansoura, Egypt \\ ${ }^{2}$ Department of Biophysics, Faculty of Medicine, Ain Shams University, Cairo, Egypt \\ ${ }^{3}$ Department of Physics, Faculty of Science, Damietta University, Egypt \\ Email: aymanstohy@yahoo.com
}

How to cite this paper: Ibrahim, A.G., Mohamed, I.E. and Zidan, H.M. (2018) Dosimetric Comparison of Amorphous Silicon EPID and 2D Array Detector for Pre-Treatment Verification of Intensity Modulated Radiation Therapy. International Journal of Medical Physics, Clinical Engineering and Radiation Oncology, 7, 438-452. https://doi.org/10.4236/ijmpcero.2018.74037

Received: June 22, 2018

Accepted: September 25, 2018

Published: September 28, 2018

Copyright $\odot 2018$ by authors and Scientific Research Publishing Inc. This work is licensed under the Creative Commons Attribution International License (CC BY 4.0).

http://creativecommons.org/licenses/by/4.0/

\begin{abstract}
Purpose: To study the dosimetric characteristics of amorphous silicon Electronic Portal Imaging Device EPID and 2D array detector for dose verification of radiotherapy treatment plans, and the quality assurance QA testing of IMRT was investigated. Materials and methods: All measurements were done with Varian IX linear accelerator, aSi-1000 EPID and 2D array detector. The dose linearity, reproducibility, output factors, dose rate, SDD and response with slap phantom thickness have been measured and compared against those measured by ion chamber. Results: The characteristics of EPID and $2 \mathrm{D}$ array: the response of EPID agreed with $2 \mathrm{D}$ array and ion chamber $0.6 \mathrm{cc}$. EPID and $2 \mathrm{D}$ array showed short-term output reproducibility with $\mathrm{SD}$ $=0.1 \%$. The dose rates of $2 \mathrm{D}$ array $\mathrm{SD}= \pm 0.7 \%$, EPID $= \pm 0.4 \%$ compared with a $0.6 \mathrm{cc} \mathrm{SD}= \pm 0.5 \%$. Output factor measurements for the central chamber of the EPID and 2D array showed no considerable deviation from ion chamber measurements. Measurement of beam profiles with the EPID and $2 \mathrm{D}$ array matched very well with the ion chamber measurements in the water phantom. The EPID is more sensitive to lower energy photons by increasing solid water phantom thickness. The mean and standard deviation passing rates $\left(\gamma_{\% \leq 1}\right)$ for film, 2D array and EPID for 30 IMRT fields of five patients were $95.93 \pm 0.96 \%, 99.05 \pm 0.24 \%$, and $99.37 \pm 0.12 \%$, respectively. Conclusion: The study shows that EPID and 2D array are a reliable and accurate dosimeter and a useful tool for quality assurance. We found that the EPID was more accurate compared with both $2 \mathrm{D}$ array and ion chamber. The gamma criterion of $3 \% / 3 \mathrm{~mm}$ is the most suitable criteria for IMRT plans of QA.
\end{abstract}




\section{Keywords}

IMRT, EPID, 2D Array Detector, Gamma Index, Quality Assurance

\section{Introduction}

The quality assurance (QA) procedure in radiotherapy generally demands dose measurement as well as patient positioning check. In conventional techniques, the dosimetric verification is based on well-tried methods carried out mostly during treatment sessions [1]. The verification of radiotherapy treatment plans is a very important step in complex radiotherapy techniques because the primary goal of radiation therapy is to deliver doses of ionizing radiation to a target volume while minimizing the dose to critical organs and healthy tissues. Ionization chamber array has become the standard device for quality assurance measurements in modern radiotherapy. In particular, the possibility of producing complex fields and dose shaping using devices such as multi leaf collimators (MLCs) has improved conformal radiotherapy techniques and boosted the clinical implementation of intensity modulated radiotherapy (IMRT) [2]. The increased complexity of clinical treatments raises the need for more accurate dose verification systems and procedures. Spezi E. et al. (2005) [3] characterized of a 2D ion chamber array for the verification of radiotherapy treatments. Markovic M.et al. (2014) [4] evaluated the Octavius Detector 1000 is an accurate, precise, and reliable detector, very useful for the daily performance of the patient specific quality assurance of radiotherapy treatment plans. EPID and 2D array have become the standard devices for QA measurements in modern radiotherapy techniques such as IMRT [5] [6]. For clinical dosimetry, the precision of dose delivery using linear accelerators to a patient must be accurate to within $\pm 2 \%$ [7] [8].

Van Esch et al. (2004) [9] evaluated the portal dose prediction model using the algorithm for amorphous silicon (a-Si) detector measurement and tested it for clinical IMRT treatment fields. IMRT is advanced form of 3D conformal radiation therapy (3DCRT); it is a powerful tool for delivery of conformal dose distributions [10]. IMRT is used in MLCs to change the intensity of the beam delivered to the tumor. As leaf motions are controlled by a computer, the IMRT technique lends itself to automated treatment delivery, eliminating the need for re-entry into the room between fields. During treatment, the leaf positions are verified by the computer, ensuring better quality control than when using customized field shaping blocks. Therefore, treatment quality assurance is necessary to define the difference between calculated and actual dose distributions [11] [12]. The implementation of IMRT in external beam therapy imposes high demands on the measurement device and quality assurance. Generally, three-dimensional dose distributions obtained from a treatment planning system have to be verified by dosimetric means. Mainly a comparison of two-dimensional calculated and measured data in several coplanar planes is performed [13] [14]. 
The dose can be measured directly, after calibration of the ionization chamber array. Though their resolution is less, compared to that of films or EPIDs, good dosimetric agreement was noticed between films and $2 \mathrm{D}$ ionization chambers for verification of radiotherapy plans, as reported by Spezi et al. [3]. To verify IMRT dose distribution, two dimensional detectors are used 2D array chamber or EPID. The properties of the EPID and 2D array chamber for pre-treatment verification of IMRT should be studied [15] [16]. Portal dose prediction (PDP) algorithm is performed in Eclipse treatment planning system (TPS) to test IMRT clinical treatment fields. The complexity of treatment needs more accurate dose verification systems and comprehensive procedures reports of QA in radiotherapy treatment planning [17] [18] [19]. Availability of new detectors with improved characteristics, better treatment calculation algorithms, modern treatment delivery machines and modes of delivery, made possible to improve on the conventional QA standards. The validity of aSi1000 EPID, 2D array detectors, and $3 \mathrm{D}$ verification systems as an ideal dosimeter for IMRT patient-specific QA [20] [21].

The gamma index (GI) evaluation used to evaluate measured distributions in detector systems against the dose distribution predicted by treatment planning system. The Gamma index (GI) results of each plan were recorded for the passing criteria, and evaluated 3\% DD, $3 \mathrm{~mm}$ DTA criteria for passing result by using EPID and 2D array detector, calculated the mean and Standard Deviations (SD) for each plan [9].

This study was carried out to evaluate the dosimetric characteristics performance of EPID and 2D array for IMRT dose verification. To quantify the performance of the device, some of the basic dosimetry tests were carried out and also some of the tests were compared with the ionization chamber measurements. The basic tests included linearity, reproducibility, output factors dependency, dose rate dependency, and sensitivity for photon beams. The measurements carried out by the 2D array and EPID devices for verification of IMRT plans are also presented, and the same was compared with the film dosimetry measurements.

\section{Materials and Methods}

A high-energy linear accelerator (Varian Clinac IX; Varian Medical Systems, Palo Alto, CA) with nominal 6 and $18 \mathrm{MV}$ photon beams has been installed, in radiation oncology department, Ain Shams University Hospital. In the present study, we applied a $300 \mathrm{MU} \mathrm{min}^{-1}$ fixed pulse rate that is used in clinical practice. Portal Vision aSi-1000 imager panel of Varian Clinac was used, with a pixel dimension and spatial resolution of $1024 \times 768$ and $0.392 \mathrm{~mm}$ per pixel, respectively. EPID is a useful tool in the QA process with good evaluation abilities, the Portal Vision Exact-Arm (Medical Systems of Varian). The linear accelerator includes an aS-1000 Portal Vision imager and comprises of an $8 \mathrm{~mm}$ thickness main plate, a thin copper slice $(1 \mathrm{~mm})$, a $0.5 \mathrm{~mm}$ phosphor film. $2 \mathrm{D}$ array de- 
tector 1500 is 1405 ion chamber matrix in a plane of field size $27 \times 27 \mathrm{~cm}^{2}$, and plane-parallel detectors are $4.4 \times 4.4 \times 3 \mathrm{~mm}^{3}$ in size, with spacing (centre-to-centre) of $7.1 \mathrm{~mm}$ for IMRT verification using the VeriSoft software enables physicists to compare radiation dose distributions in IMRT verification plan with those calculated by TPS. PTW Farmer chamber $\left(0.6 \mathrm{~cm}^{3}\right)$ is the chamber to measure the absolute photon and electron dosimetry used in solid-state material. The pinpoint chamber is ideal for dose measurements in small fields. It's a small-sized sensitive volume of $0.015 \mathrm{~cm}^{3}$ and $2 \mathrm{~mm}$ in diameter, vented to air. Very high spatial resolution when used for scans perpendicular to the chamber axis. The slab phantom (RW3) consists of 33 plates machined to $30 \times$ $30 \mathrm{~cm}^{2}$ of various thickness. The mass density of RW3 is $1.045 \mathrm{~g} \cdot \mathrm{cm}^{-3}$ and the electron density has a factor relative to water of 1.012. The phantoms are used for monitor calibration and QA measurements, dose is measured by varying the measuring depth.

All measurements were performed on amorphous silicon aSi-1000 EPID, 2D array 1500, waterproof chamber and IX accelerator (Varian) with nominal 6 and $18 \mathrm{MV}$ energy photon beams. In this process a fixed pulse rate of $300 \mathrm{MU} \mathrm{\textrm {min } ^ { - 1 }}$ is used which the pulse rate used in clinical practice.

\subsection{EBID, 2D Array Detector and QA Characterization}

The properties of $2 \mathrm{D}$ array and electronic portal imaging device are verified in this study. The 2D Array 1500 measurement setup used throughout this process is shown in Figure 1. For the build-up and the backscatter material, a set of RW3 polystyrene plates (PTW, Freiburg, Germany) are used. The build-up of thickness is $4.5 \mathrm{~cm}$, whereas the backscatter material is $5.0 \mathrm{~cm}$ thick. The source to surface distance (SSD) equals $95.0 \mathrm{~cm}$. The $2 \mathrm{D}$ array reference point of measurement is located at $100 \mathrm{~cm}$ from source of radiation. EPID Varian aS-1000 (Varian Medical Systems), which are routinely used in our clinic for set-up verification, was investigated in this study.

1) Dose Linearity: To verify the linear response with dose, detectors are irradiated with dose setting of 2, 5, 10, 20, 50, 100, 150, 200, 250, 300 and 500 MUs (monitor Units). The responses were compared with the measurements of $0.6 \mathrm{cc}$, EPID and 2D array. 2D array in the standard setup as shown in Figure 1, EPID is placed at a fixed detector distance of $100 \mathrm{~cm}$, field size of $10 \times 10 \mathrm{~cm}^{2}$ centered at the central axis. The collimator and the gantry angles are both $0^{\circ}$. A dose of $100 \mathrm{MU}$ was delivered, integrated image was acquired for $6 \mathrm{MV}$ photon with dose rates of $100 \mathrm{MU} / \mathrm{min}$ to $600 \mathrm{MU} / \mathrm{min}$.

2) Dose Reproducibility: The reproducibility is the \% difference between successive measurements for the same radiation dose. The performance of EPID and 2D array in standard setup Figure 1 were measured to verify short-term, over a period of ten days. The output was measured by delivering $100 \mathrm{MU}$ for a fixed field size of $10 \times 10 \mathrm{~cm}^{2}$ at SDD $100 \mathrm{~cm}$ using photon energy $6 \mathrm{MV}$. The measurement was repeated for 10 readings. 


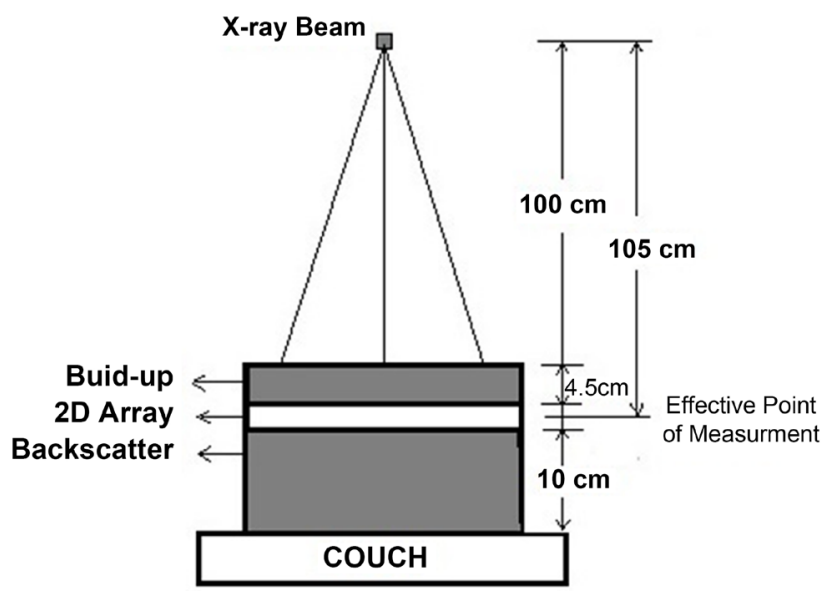

Figure 1. The $2 \mathrm{D}$ array setup at standard measurement. The array is put between a build-up and backscatter material.

3) Dose rate dependency: The dose rate response of EPID and 2D array in standard setup Figure 1 is compared with $0.6 \mathrm{cc}$ ion chamber using photon energy $6 \mathrm{MV}$. The detector was irradiated by delivering $200 \mathrm{cGy}$ for a $10 \times 10$ $\mathrm{cm}^{2}$ field size at various dose rates $(100,200,300,400,500 \& 600 \mathrm{MU} / \mathrm{min})$ at a fixed distance of $100 \mathrm{~cm}$.

4) Field size dependence: Field size response of the EPID and 2D array in standard setup Figure 1 were evaluated in comparison with pinpoint detector (type 31014). The pinpoint detector was connected to electrometer (PTW, Freiburg, Germany). Dose outputs were compared with pinpoint ion chamber measurements at the same conditions using an RW3 plate. All measurements were corrected for temperature and pressure, by delivering $100 \mathrm{MU}$ for the field sizes of $2 \times 2 \mathrm{~cm}^{2}$ to $25 \times 25 \mathrm{~cm}^{2}$. The field size response of the EPID was compared to $2 \mathrm{D}$ array measurement. The detector was positioned at $100 \mathrm{~cm}$ from the source and field sizes. Both sets of measurements were normalized to the $10 \times 10 \mathrm{~cm}^{2}$ values.

5) SDD dependency: The SDD dependency was studied for $6 \mathrm{MV}$ and $18 \mathrm{MV}$ photon beams to evaluate the effect of SDD on EPID and 2D array in standard setup Figure 1, the detectors were irradiated with $100 \mathrm{MU}$ and $10 \times 10 \mathrm{~cm}^{2}$ field size, the doses were measured for different source to detector distance SDD of $90,95,100,105,110,115,120 \mathrm{~cm}$. The measured values were plotted against the varying Source to Detector Distance (SDD).

6) Verification of response with slap phantom thickness. Intensity verification of a photon beam is reduced by increasing the thickness of RW3 slab phantom with EPID or 2D array, each phantom is positioned on the beam central axis of the treatment couch and the distance from the source to the centre of the phantom SAD is $100 \mathrm{~cm}$. Figure 2 shows the setup used thicknesses of 5, 10, 15, 20, 25 and $30 \mathrm{~cm}$. The transit signals through the phantoms were detected either directly with the EPID (Figure 2(a)) or using a 2D array positioned on the central axis at the EPID position (Figure 2(b)). For the EPID, the response was 


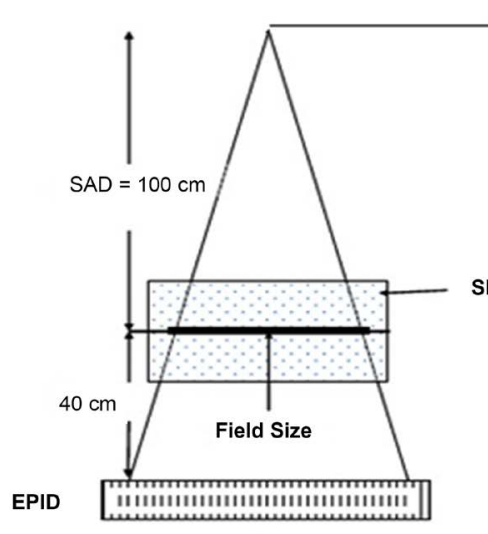

(a)

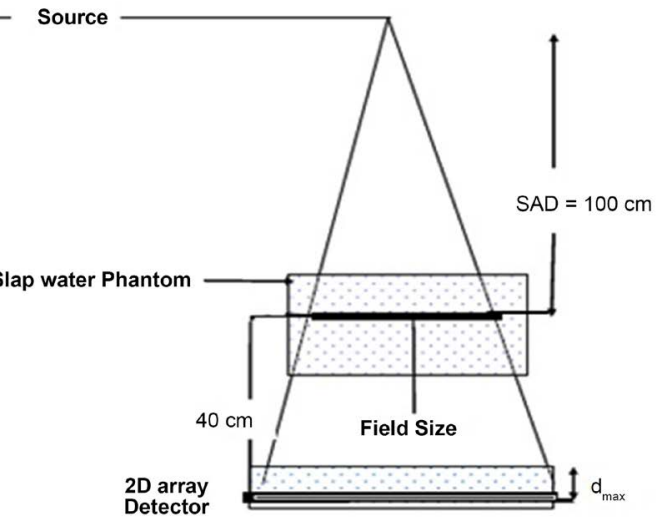

(b)

Figure 2. The experimental setups for (a) EPID and (b) 2D array measurement of scatter and response with increased thickness. The solid water phantom thickness was symmetrically set in the isocentre and the detectors kept at fixed source to detector distance (SDD $=140 \mathrm{~cm}$ ).

measured as the mean pixel value in a $1 \mathrm{~cm}$ region of interest in the centre of the image. For the alternative method, the $2 \mathrm{D}$ array was inserted in a $30 \times 30 \mathrm{~cm}^{2}$ solid water phantom slab, with appropriate build-up $(1.5 \mathrm{~cm}$ for $6 \mathrm{MV})$ and 1 $\mathrm{cm}$ solid water phantom for back scatter to simulate the EPID.

\subsection{IMRT Dose Distribution Verification}

In this study, IMRT QA with film, 2D array and EPID were used in the gamma index method to compare calculated TPS dose with measured dose, using 3\% 3 $\mathrm{mm}$ gamma criteria. The traditional method of QA for IMRT was 2Dimensional testing using film (Kodak X-OMAT). The dose was measured at source to axis distance $(\mathrm{SAD})$ of $100 \mathrm{~cm}$, with the film located at $10 \mathrm{~cm}$ depth of slab phantom and a gantry angle of $0^{\circ}$. Similar to film QA, the dose is measured at the standard measurement of setup for the $2 \mathrm{D}$ array at a gantry angle of $0^{\circ}$. For EPID, SAD was set at $100 \mathrm{~cm}$ and at a gantry angle of $0^{\circ}$. Measurement was done by EPID without phantom and EPID dedicated software (Eclipse, Ver. 8.9, Varian Medical System, and USA) was used to verify dose delivery after the beam measurement. IMRT was used for three (two males and one female) head and neck $(\mathrm{H} \& \mathrm{~N})$ plans, one male cervical spine and one male pelvises were selected to evaluate the mean and standard deviations (SD) of gamma index. The Gamma evaluations (DD and DTA) of measured dose against TPS calculated doses were performed for 25 IMRT cases (177 Fields). All the cases were planned in Eclipse treatment planning system and the QA plans for absolute point dose measurements, portal dosimetry, and 2D array were created for the TPS calculated planar dose distributions. The calculated and measured dose for each plan was compared on the basis of $3 \% 3 \mathrm{~mm}$ gamma criteria (DD and DTA). For the portal dosimetry, area gamma $>1 \%$, average gamma, and maximum gamma were measured and tabulated. For the $2 \mathrm{D}$ array, the percentage of the pixels passed the acceptance criteria $3 \% 3 \mathrm{~mm}$ were calculated and tabulated. The mean and 
standard deviation for all the gamma parameters were calculated and compared. The criteria validity accepted as section with gamma value $\gamma_{0 \leq 1}=95 \%$. Gamma parameters, $\gamma_{\max }, \gamma_{\text {avg }}$ and $\gamma_{\% \leq 1}$ were estimated for each field and calculated the mean and standard deviations (SD).

\section{Results and Discussion}

\subsection{EBID, 2D Array Detector and QA Characterization}

The characteristics of EPID and 2D array detectors have been investigated for periodic QA applications. Study verification of characteristics linearity, Reproducibility, dose rate, Field size, SSD, and response with slap phantom thickness response for both 2D array detector and EPID.

1) Dose Linearity: The comparison of measured values for different monitor units ranging 2 to $500 \mathrm{MU}$ were analyzed for $0.6 \mathrm{cc}$ ion chamber, 2D array and EPID at field size $10 \times 10 \mathrm{~cm}^{2}$. The linearity result of $2 \mathrm{D}$ array and EPID were compared with ion chamber $0.6 \mathrm{cc}$ as shown in Figure 3. A linear coefficient of determination $(y=0.889 x-2.1218), R^{2}=0.9991$ for EPID, also for $2 \mathrm{D}$ array $(y=0.9428 x+1.5358), \quad R^{2}=0.9989$ and $0.6 \mathrm{cc}$ ion chamber $(y=0.9554 x+2.3346), \quad R^{2}=0.9989$. The response of EPID was the best, compared to both the $2 \mathrm{D}$ array and $0.6 \mathrm{cc}$ ion chamber. To compare these outcome measures between the three methods, one-way-ANOVA and Tukey's honest significant difference tests were employed. A significance level of $5 \%$ was considered. We observed the difference between the methods was statistically significant $(\mathrm{p}=0.002)$.

2) Dose Reproducibility: The reproducibility of the measurements within each set was excellent short-term stability as shown in the Figure 4. Response

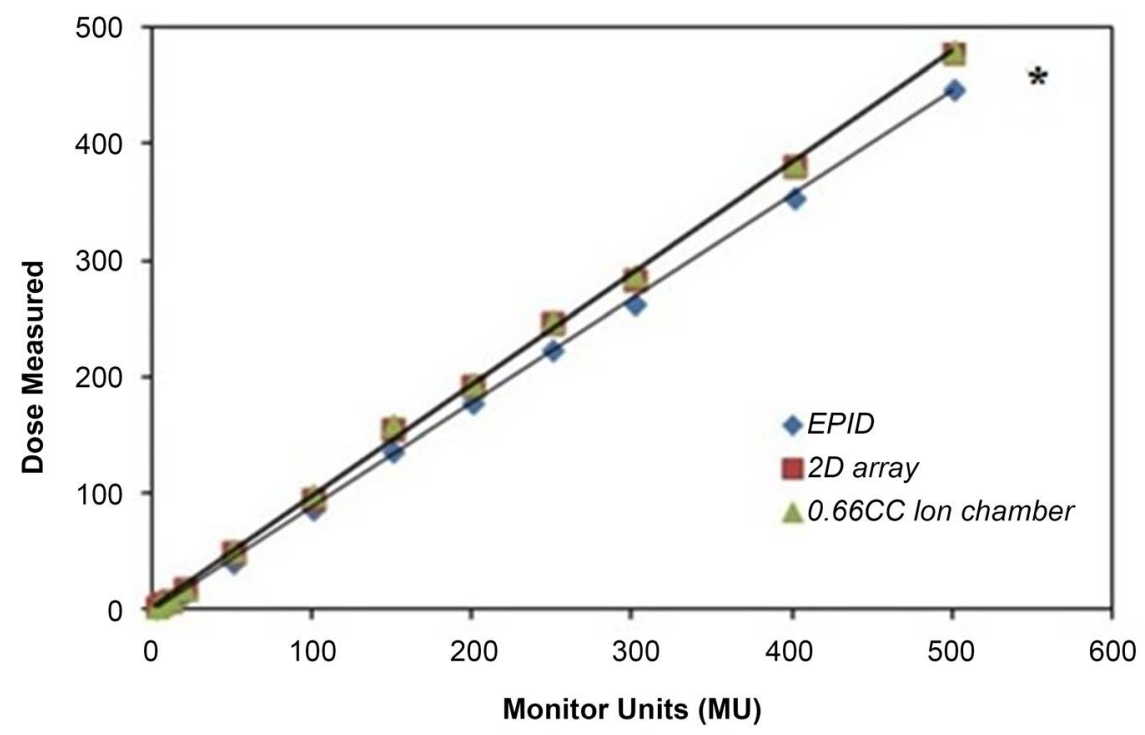

Figure 3. Linearity test for $6 \mathrm{MV}$ photon beam by irradiating the EPID, $2 \mathrm{D}$ array and 0.6 cc ion chamber with a field size $10 \times 10 \mathrm{~cm}^{2}$, at $100 \mathrm{~cm}$, gantry angle $0^{\circ} .\left(^{*}\right)$ the mean difference is significant at the 0.05 level. 


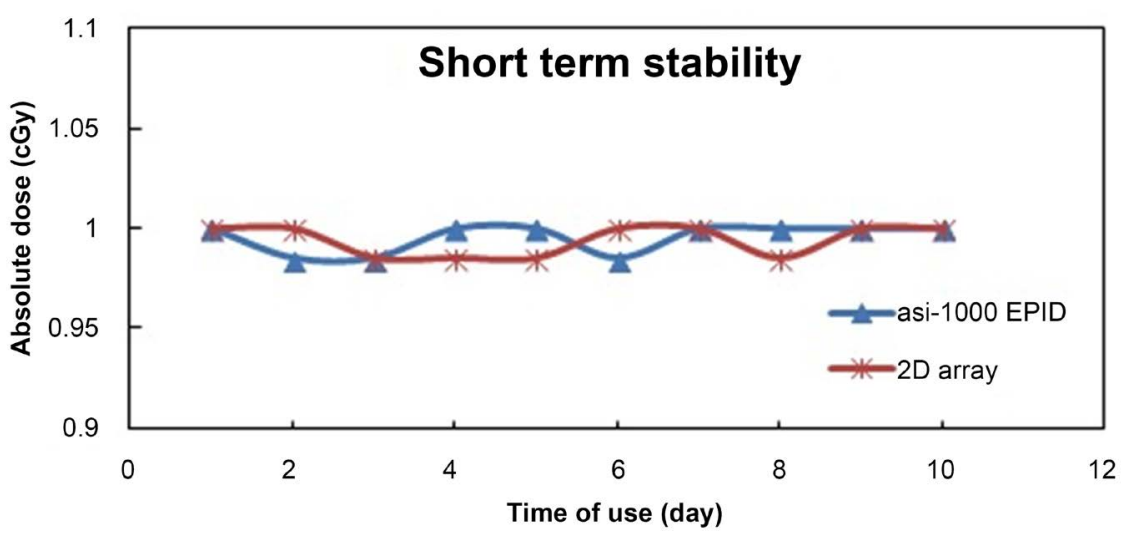

Figure 4. EPID and 2D array, the irradiation was repeated ten times using energy $6 \mathrm{MV}$ over a period of short-term.

variation from chamber to chamber was $\pm 1 \%$. The EPID and $2 \mathrm{D}$ array demonstrates excellent short-term output reproducibility with a maximum standard deviation of $0.1 \%$.

3) Dose rate dependency: The response of dose rate for EPID and 2D array compared to $0.6 \mathrm{cc}$ ion chamber measurements for $6 \mathrm{MV}$ photon energy, at $10 \times$ $10 \mathrm{~cm}^{2}$ field size at various dose rates $(100,200,300,400,500 \& 600 \mathrm{MU} / \mathrm{min})$ at a fixed distance of $100 \mathrm{~cm}$ as shown in Figure 5 . The detector panel did not display any significant dose rate dependant saturation in response with the dose rate range $100 \mathrm{MU} / \mathrm{min}$ to $600 \mathrm{MU} / \mathrm{min}(< \pm 0.5 \%)$. The results showed that the $2 \mathrm{D}$ array had high dose rate independent response for the dose rates ranging from $100-600 \mathrm{MU} / \mathrm{min}$ with SD of $\pm 0.7 \%$ for $6 \mathrm{MV}$ and SD of $\mathrm{EPID}= \pm 0.4$. The results were compared with a $0.6 \mathrm{cc}$ chamber with a maximum variation of $\mathrm{SD}= \pm 0.5 \%$ with $6 \mathrm{MV}$.

4) Field size dependence: The output factor measurement results are shown in Figure 6, the field size dependent output factor curve of EPID, 2D array and pinpoint detector for $6 \mathrm{MV}$ photon energy. The compatibility between EPID, 2D Array values and the pinpoint dataset was very good. The variation for EPID and $2 \mathrm{D}$ array measurements with pinpoint was found to be $\pm 0.5 \%$ difference for all field size. The EPID and 2D array readings with field size were normalized to 10 $\times 10 \mathrm{~cm}^{2}$ values as shown in Figure 7. The EPID signal was divided by the 2D array measurements. The EPID pixel values for the three separate irradiations were less than $0.1 \%$ standard deviation.

5) $S D D$ dependency: The responses of detectors of SDD for $6 \mathrm{MV}$ and 18 MV photon beams are displayed in Figure 8, the results were compared to those obtained using a 2D array for the same measurement setup. Both EPID and 2D array measurement were within $1 \%$ for the range of SDDs performed in this study. The mean and standard deviation for $6 \mathrm{MV}$ and $18 \mathrm{MV}$ photon energy $($ mean $\pm S D)$ value $=(1.96 \pm 0.987 \%),(1.39 \pm 0.452 \%)$ respectively.

6) Verification of response with slap phantom thickness. The intensity of a photon beam was reduced as the absorbing material thickness was increased. 


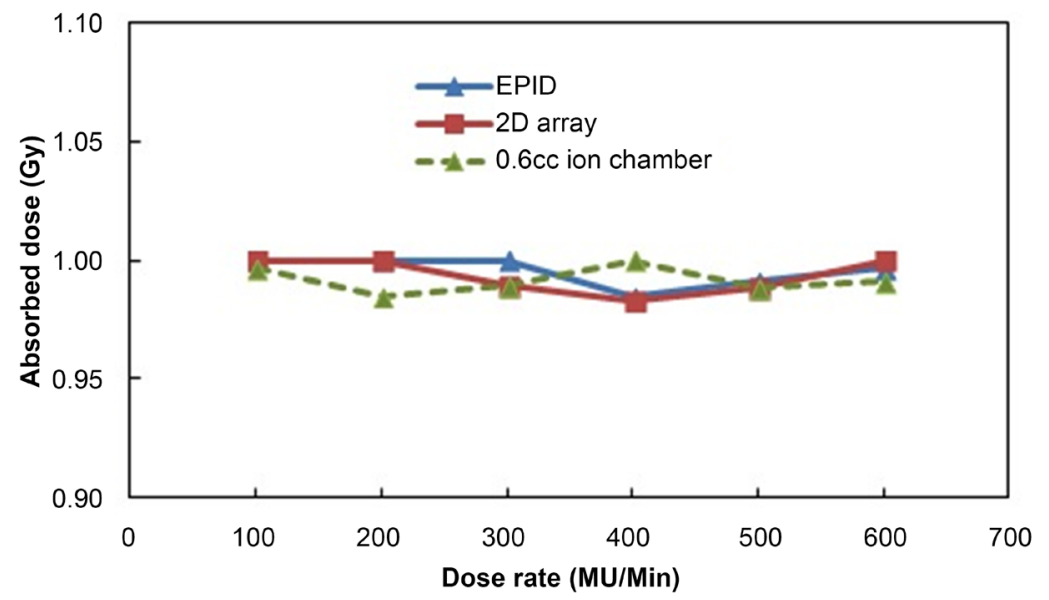

Figure 5. Dose rate response curve for 6-MV photon energy.

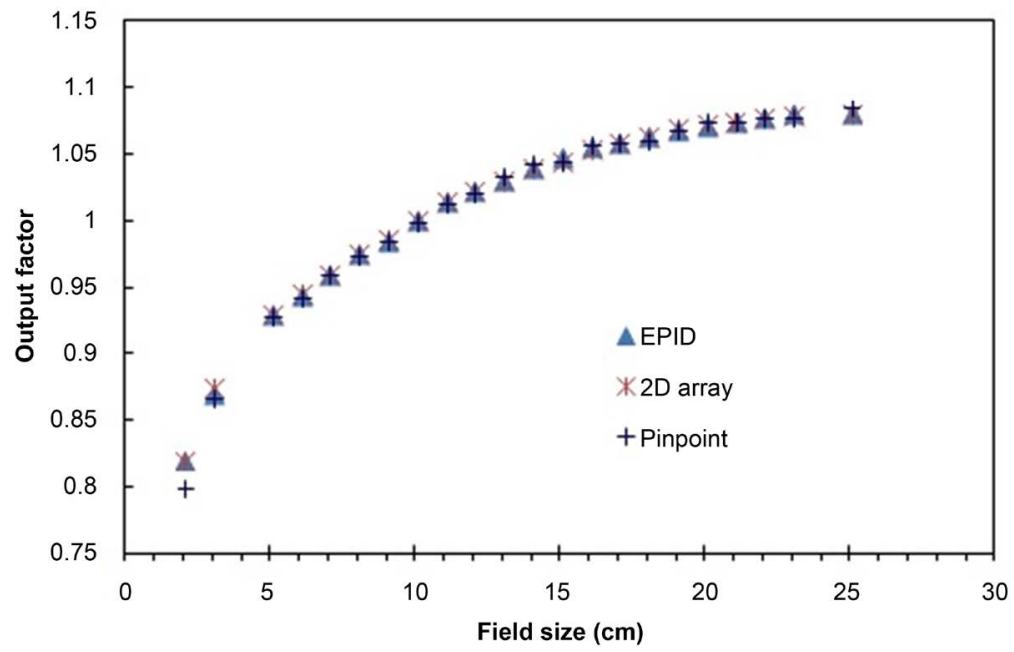

Figure 6. Output factor of EPID and 2D array comparison with pinpoint chamber for 6 $\mathrm{MV}$ and photons at the same condition. Field sizes range between $2 \times 2 \mathrm{~cm}^{2}$ and $25 \times 25$ $\mathrm{cm}^{2}$.

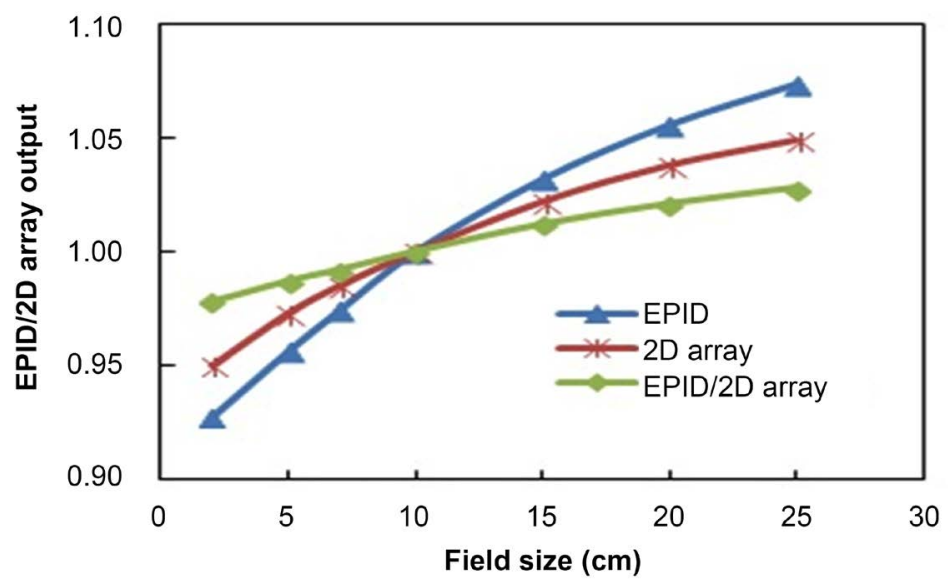

Figure 7. The EPID signal change with field size is compared to the change in dose with field size measured by $2 \mathrm{D}$ array. The data are normalized to the $10 \times 10 \mathrm{~cm}^{2}$ field. 


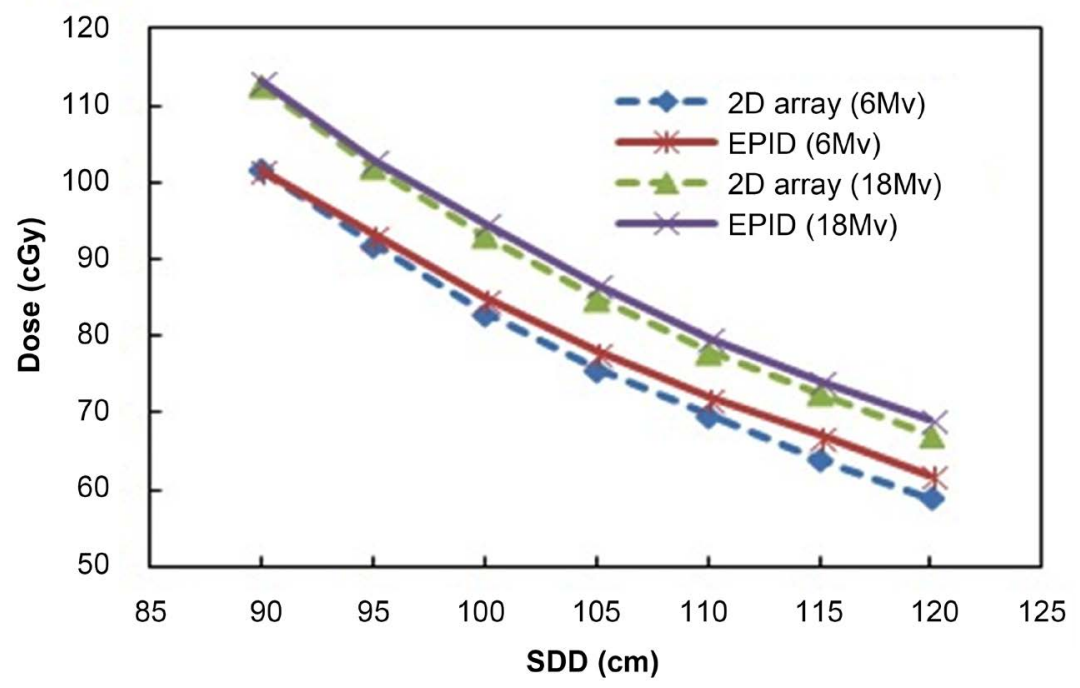

Figure 8. SDD response curve for $6 \mathrm{MV}$ and $18 \mathrm{MV}$. 2D array values have been compared with EPID measurements.

Figure 9 shows the reduction in photon intensity measured with the EPID and $2 \mathrm{D}$ array responses when solid water phantom materials of various thicknesses were placed between the source and the detectors (EPID and 2D array). At each phantom thickness, both detectors were exposed at the same radiation conditions. Both the EPID and 2D array signals were acquired on the central axis, and the data for each detector was normalized to those when there was no absorbing material (thickness $=$ zero). By increasing the solid water thickness, the EPID was more sensitive to lower energy photons, its response decreased more rapidly than the $2 \mathrm{D}$ array as a function of attenuator thickness.

Both the detector system showed good response for IMRT patient specific QA. The EPID field verification could be done very effectively with an excellent spatial resolution. The disadvantages of the $2 \mathrm{D}$ array system are: the low resolution of the detectors, the time taken to set up the detectors, phantom and connect to the external computer system with analysis software. Syamkumar S. A. et al. (2012) [2] reported, the 2D array provides an overall accuracy when compared with single ionization chamber measurements for static and rotational delivery. Moreover, the dose calibration for the 2D array is easy and stable. 2D array and EPID are the tool for the quality assurance and the verification of radiotherapy plans.

\subsection{IMRT Dose Distribution Verification}

The Dose was calculated using TPS compared with dose measured by the dosimetric tools based on gamma evaluation $(3 \% / 3 \mathrm{~mm})$. Table 1 shows the mean passing rates of gamma index, for the treatment fields of each patient using film, 2D array, and EPID. The values measured with dosimetry tools corresponded with the calculated values for five patients. The mean and standard deviation (mean \pm SD) passing rates $\left(\gamma_{\% \leq 1}\right)$ for film, 2D array and EPID for 30 IMRT fields 


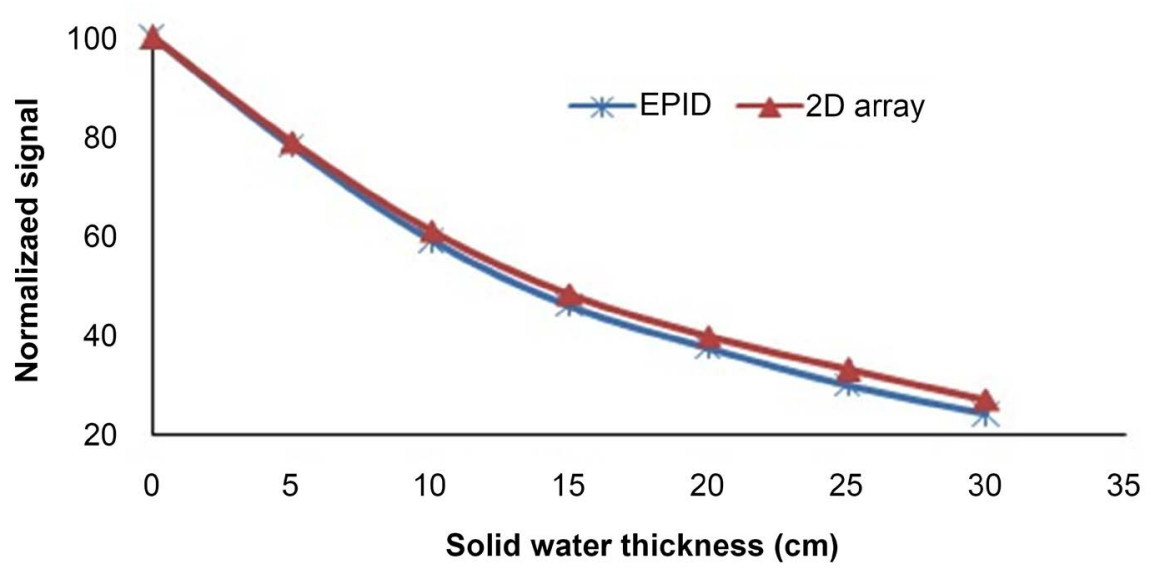

Figure 9. EPID versus 2D array response with solid water thickness Increasing.

Table 1. Mean passing rates based on the gamma index method for the treatment fields of each patient using film, 2D array, and electronic portal imaging device (EPID).

\begin{tabular}{ccccccc}
\hline Dosimetry tools & Patient 1 & Patient 2 & Patient 3 & Patient 4 & Patient 5 & Mean \pm SD \\
\hline Film & 97.41 & 95.45 & 96.03 & 94.82 & 95.95 & $95.93 \pm 0.96$ \\
2D array & 99.12 & 99.28 & 98.74 & 98.87 & 99.26 & $99.05 \pm 0.24$ \\
EPID & 99.43 & 99.22 & 99.32 & 99.54 & 99.34 & $99.37 \pm 0.12$ \\
\hline
\end{tabular}

of five patients were $95.93 \% \pm 0.96 \%, 99.05 \% \pm 0.24 \%$, and $99.37 \% \pm 0.12 \%$, respectively. All acceptable passing rate of $95 \%$, these tools showed some differences in measuring the same beam, with the gamma index being much lower for film than for the other tools. This comparison of gamma indices for film, 2D array, and EPID showed differences in dose distribution when using various dosimetric tools to carry out the QA for the same patients IMRT, results of QA dependency on dosimetric tools. We found that the EPID was more accurate compared with both $2 \mathrm{D}$ array and film.

A dosimetric IMRT field verification of 177 IMRT fields was carried out with $2 \mathrm{D}$ array detector by comparing the measured dose distribution to portal dosimetry measurement and TPS calculations. The results of gamma evaluation for 25 cases were tabulated as shown in the Table 2 , the data of $3 \% \mathrm{DD}$, and $3 \mathrm{~mm}$ DTA passing criteria of dose distribution using portal dosimetry and 2D array detector of various tumours. The mean and standard deviation (SD) of $\gamma_{\% \leq 1}, \gamma_{\max }$ and $\gamma_{\text {avg }}$ estimated from all fields of each IMRT plan, using EPID and 2D array detector, PDP predicted and EPID measured photon dose distribution corresponded with mean \pm SD value for $\gamma_{\% \leq 1}=99.01 \% \pm 0.74 \%, \gamma_{\max }=2.10 \% \pm 0.57 \%$, and $\gamma_{\text {avg }}=0.23 \% \pm 0.04 \%$, respectively. Independent verification of the planned dose from the same IMRT fields using 2D array detector also resulted in comparable values of $\gamma_{\% \leq 1}=98.06 \% \pm 0.75 \%, \gamma_{\max }=1.66 \% \pm 0.45 \%$ and $\gamma_{\text {avg }}=0.27 \% \pm$ $0.04 \%$.

Studies of dosimetric characteristics are essential before using at all dosimetric tools for the clinical purpose. At the present time portal dosimetry and 2D array 
Table 2. Showing data of 3\% DD, $3 \mathrm{~mm}$ DTA passing criteria of dose distribution using portal dosimetry and 2D array for 177 fields of various tumours.

\begin{tabular}{|c|c|c|c|c|c|c|c|}
\hline \multirow{2}{*}{$\begin{array}{c}\text { Patient } \\
\text { No. }\end{array}$} & \multirow{2}{*}{ Site } & \multicolumn{3}{|c|}{ Portal Dosimetry } & \multicolumn{3}{|c|}{$2 \mathrm{D}$ array } \\
\hline & & $\gamma_{\% \leq 1}(\%)$ & $\gamma_{\max }$ & $\gamma_{\text {avg }}$ & $\gamma_{\% \leq 1}(\%)$ & $\gamma_{\max }$ & $\gamma_{\text {avg }}$ \\
\hline 1 & Head \& Neck & 98.1 & 3.35 & 0.24 & 97.62 & 2.95 & 0.26 \\
\hline 2 & Head \& Neck & 97.8 & 2.53 & 0.26 & 96.82 & 2.42 & 0.28 \\
\hline 3 & Head \& Neck & 99.4 & 1.66 & 0.21 & 97.84 & 1.31 & 0.25 \\
\hline 4 & Head \& Neck & 97.9 & 1.42 & 0.22 & 98.29 & 1.44 & 0.31 \\
\hline 5 & Pelvis & 99.5 & 1.47 & 0.25 & 97.37 & 1.39 & 0.29 \\
\hline 6 & Pelvis & 99.4 & 1.71 & 0.24 & 98.55 & 1.58 & 0.34 \\
\hline 7 & Pelvis & 99.1 & 1.49 & 0.35 & 97.1 & 1.62 & 0.27 \\
\hline 8 & Pelvis & 98.7 & 1.21 & 0.23 & 97.4 & 1.16 & 0.23 \\
\hline 9 & Head \& Neck & 98 & 3.35 & 0.29 & 98.83 & 2.76 & 0.32 \\
\hline 10 & Head \& Neck & 99.2 & 2.71 & 0.21 & 97.61 & 1.83 & 0.27 \\
\hline 11 & Pelvis & 100 & 1.81 & 0.18 & 98.74 & 1.24 & 0.23 \\
\hline 12 & cervical spine & 98.5 & 2.15 & 0.28 & 97.83 & 1.31 & 0.28 \\
\hline 13 & Head \& Neck & 98.7 & 2.52 & 0.27 & 97.52 & 1.37 & 0.32 \\
\hline 14 & Head \& Neck & 99.4 & 1.91 & 0.25 & 97.43 & 1.69 & 0.29 \\
\hline 15 & Head \& Neck & 99.8 & 1.94 & 0.27 & 98.4 & 1.39 & 0.24 \\
\hline 16 & Pelvis & 99.2 & 2.6 & 0.19 & 98.35 & 1.85 & 0.23 \\
\hline 17 & Pelvis & 99.9 & 1.8 & 0.23 & 97.53 & 1.58 & 0.21 \\
\hline 18 & Pelvis & 99.9 & 1.49 & 0.21 & 98.75 & 1.32 & 0.29 \\
\hline 19 & Head \& Neck & 98.4 & 2.15 & 0.22 & 97.46 & 1.61 & 0.25 \\
\hline 20 & Pelvis & 97.9 & 2.73 & 0.26 & 98.21 & 1.89 & 0.32 \\
\hline 21 & Pelvis & 99.7 & 2.57 & 0.18 & 97.52 & 1.79 & 0.26 \\
\hline 22 & Pelvis & 99.9 & 2.1 & 0.2 & 98.87 & 1.37 & 0.19 \\
\hline 23 & Pelvis & 99.8 & 1.85 & 0.17 & 99.68 & 1.81 & 0.24 \\
\hline 24 & Pelvis & 98.5 & 1.92 & 0.21 & 99.74 & 1.38 & 0.26 \\
\hline 25 & Pelvis & 98.5 & 2.02 & 0.22 & 98.12 & 1.42 & 0.32 \\
\hline & Mean & 99.01 & 2.10 & 0.23 & 98.06 & 1.66 & 0.27 \\
\hline & SD & 0.74 & 0.57 & 0.04 & 0.75 & 0.45 & 0.04 \\
\hline
\end{tabular}

detector verification systems are adopted for the patient specific QA due to excellent dosimetric characteristics and easiness to use. Dosimetric properties of aSi1000 EPID and 2D array system proved its worth over film and other dosimetric system. The dosimetric characteristics are required for the development of an effective and efficient algorithm and dosimetric measurement tool for the better accuracy. Both the detector system showed good response for IMRT and VMAT patient specific QA. With the introduction of aSi1000 EPID individual field verification can be done very effectively with an excellent spatial resolution. The disadvantages of the 2D array system are the low resolution of the detectors 
and the time taken to setup the detectors and phantom connect with the external computer system with analysis software. The values obtained with the portal dosimetry system were found to be relatively more consistent compared to those obtained with $2 \mathrm{D}$ array detector system. For pretreatment verification of IMRT plans have been carried out using the $2 \mathrm{D}$ array and verification by EPID dose measurement, the passing criteria for IMRT plans was based on the percent of pixels passing gamma $>95 \%$ within the passing criteria of dose difference (DD) $3 \%$, distance to agreement (DTA) $3 \mathrm{~mm}$ DTA. The result shows an agreement between the measurement by the EPID and 2D array. Every point measured in these plans agreed to within $\pm 5 \%$ acceptability criteria of the dose calculated by the planning system and the chamber measured dose.

\section{Conclusion}

The results showed that both of 2D array and EPID can be used in patient specific QA measurements for IMRT. It is a useful tool for the quality assurance and the verification of radiotherapy plans. The $2 \mathrm{D}$ array provides an overall accuracy when compared with single ionization chamber measurements for IMRT delivery. Moreover, the dose calibration for the $2 \mathrm{D}$ array is easy and stable. But EPID is more accurate dosimeter and a useful tool for quality assurance. The EPID of IMRT patient specific QA is great potential for saving time and for the verification of individual IMRT fields. The disadvantages of the $2 \mathrm{D}$ array system are: the low resolution of the detectors, the time taken to set up the detectors, phantom and connect to the external computer system with analysis software. The results showed that the gamma criterion of $3 \% / 3 \mathrm{~mm}$ is the most suitable criteria for IMRT plans QA. The result shows a very good agreement between measured dose and calculated dose of the TPS, proving that our treatment planning using patient specific IMRT QA is the sufficient practice for IMRT treatment.

\section{Conflicts of Interest}

The authors declare no conflicts of interest regarding the publication of this paper.

\section{References}

[1] Grzadziel, A., Smolińska, B., Rutkowski, R. and Ślosarek, K. (2007) EPID Dosimetry-Configuration and Pre-Treatment IMRT Verification. Reports of Practical Oncology and Radiotherapy, 12, 307-312. https://doi.org/10.1016/S1507-1367(10)60069-7

[2] Syamkumar, S.A., Padmanabhan, S., Sukumar, P. and Nagarajan, V. (2012) Characterization of Responses of 2d Array Seven 29 Detector and Its Combined Use with Octavius Phantom for the Patient-Specific Quality Assurance in Rapidarc Treatment Delivery. Medical Dosimetry, 37, 53-60. https://doi.org/10.1016/j.meddos.2010.12.013

[3] Spezi, E., Angelini, A.L., Romani, F. and Ferri, A. (2005) Characterization of a 2D 
Ion Chamber Array for the Verification of Radiotherapy Treatments. Physics in Medicine \& Biology, 50, 3361-3373. https://doi.org/10.1088/0031-9155/50/14/012

[4] Markovic, M., Stathakis, S., Mavroidis, P., Jurkovic, I. and Papanikolaou, N. (2014) Characterization of a Two-Dimensional Liquid-Filled Ion Chamber Detector Array Used for Verification of the Treatments in Radiotherapy. Medical Physics, 41, Article ID: 051704/14. https://doi.org/10.1118/1.4870439

[5] Sharma, D.S., Mhatre, V., Heigrujam, M., Talapatra, K. and Mallik, S. (2010) Portal Dosimetry for Pretreatment Verification of IMRT Plan: A Comparison with 2D Ion Chamber Array. Journal of Applied Clinical Medical Physics, 11, 238-248. https://doi.org/10.1120/jacmp.v11i4.3268

[6] Ezzel, G.A., Galvin, J.M., Low, D., Palta, J.R., Rosen, I. and Sharpe, M.B. (2003) Guidance on Delivery, Treatment Planning, and Clinical Implementation of IMRT: Report of the IMRT Subcommittee of the AAPM Radiation Therapy Committee. Medical Physics, 30, 2089-2115. https://doi.org/10.1118/1.1591194

[7] Sun, W., Ren, C., Lu, N., Xu, Y., Han, F., Ping, L.Y. and Dai, J. (2016) Dosimetric Comparison of Intensity-Modulated Radiotherapy and Volumetric-Modulated Arc Radiotherapy in Patients with Prostate Cancer: A Meta-Analysis. Journal of Applied Clinical Medical Physics, 17, 254-262. https://doi.org/10.1120/jacmp.v17i6.6464

[8] Levendag, P.C. (2005) Dosimetric Verification of Intensity Modulated Radiotherapy with an Electronic Portal Imaging Device. Department of Radiation Oncology, Division of Medical Physics, Erasmus MC/Daniel den Hoed Cancer Center, Rotterdam.

[9] Van Esch, A., Depuydt, T. and Huyskens, D.P. (2004) The Use of an aSi-Based EPID for Routine Absolute Dosimetric Pre-Treatment Verification of Dynamic IMRT Fields, Radiation. Oncology, 71, 223-234. https://doi.org/10.1016/j.radonc.2004.02.018

[10] Khan, F.M. (2003) The Physics of Radiation Therapy. 3rd Edition, Williams \& Wilkins, Philadelphia.

[11] SGSMP, SSRPM and SSRFM (2007) Quality Control for Intensity-Modulated Radiation Therapy, Recommendations No. 15.

[12] Venencia, C.D. and Besa, P. (2004) Commissioning and Quality Assurance for Intensity Modulated Radiotherapy with Dynamic Multileaf Collimator: Experience of the Pontificia Universidad Catolica de Chle. Journal of Applied Clinical Medical Physics, 5, 37-54.

[13] Herzen, J., Todorovic, M. and Cermers, F. (2007) Dosimetric Evaluation of a 2D Pixel Ionization Chamber for Implementation in Clinical Routine. Physics in Medicine \& Biology, 52, 1197-1208. https://doi.org/10.1088/0031-9155/52/4/023

[14] Saminathan, S., Manickan, R., Chandraraj, V. and Supe, S.S. (2010) Dosimetric Study of 2D Ion Chamber Array Matrix for the Modern Radiotherapy Treatment Verification. Journal of Applied Clinical Medical Physics, 11, 3076. https://doi.org/10.1120/jacmp.v11i2.3076

[15] Peters, S., Schiefer, H. and Plasswilm, L. (2014) A Treatment Planning Study Comparing Elekta VMAT and Fixed Field IMRT Using the Varian Treatment Planning System Eclipse. Radiation Oncology, 9, 153. https://doi.org/10.1186/1748-717X-9-153

[16] Merheb, C., Chevillard, C., Ksouri, W., Fawzi, M., Bollet, M. and Toledano, A. (2015) Comparison between Two Different Algorithms Used for Pretreatment QA via aSi Portal Images. Journal of Applied Clinical Medical Physics, 16, 141-153. https://doi.org/10.1120/jacmp.v16i3.5202 
[17] Boehmer, D., Bohsung, J., Eichwurzel, I., Moys, A. and Budach, V. (2004) Clinical and Physical Quality Assurance for Intensity Modulated Radiotherapy of Prostate Cancer. Radiation Oncology, 71, 319-325.

https://doi.org/10.1016/j.radonc.2004.02.016

[18] Warkentin, B., Steciw, S., Rathee, S. and Fallone, B.G. (2003) Dosimetric IMRT Verification with a Flat-Panel EPID. Medical Physics, 30, 3143-3155.

https://doi.org/10.1118/1.1625440

[19] Greer, P.B. and Barnes, M.P. (2007) Investigation of Amorphous Silicon EPID for Measurement and Quality Assurance of Enhanced Dynamic Wedge. Physics in Medicine and Biology, 52, 1075-1087. https://doi.org/10.1088/0031-9155/52/4/014

[20] Budgell, G.J., Zhang, Q., Trouncer, R.J. and Mackay, R.I. (2005) Improving IMRT Quality Control Efficiency Using an Amorphous Silicon Electronic Portal Imager. Medical Physics, 32, 3267-3278. https://doi.org/10.1118/1.2074227

[21] Agazaryan, N., Timothy, D.S. and Demarco, J.J. (2003) Patient Specific Quality Assurance for the Delivery of Intensity Modulated Radiotherapy. Journal of Applied Clinical Medical Physics, 4, 40-50. https://doi.org/10.1120/jacmp.v4i1.2540

\section{Abbreviations and Acronyms:}

\begin{tabular}{cc}
\hline $2 D$ & Two dimension \\
3DCRT & 3D Conformal Radiation Therapy \\
$\gamma_{\% \leq 1}$ & Area Gamma \\
$\gamma_{\max }$ & Maximum Gamma \\
$\gamma_{\text {avg }}$ & Average Gamma \\
aSi-1000 EPID & amorphous silicon 1000 Electronic Portal Imaging Device \\
DD & Dose Difference \\
DTA & Distance To Agreement \\
GI & Gamma Index \\
MLCs & Multi Leaf Collimators \\
MU & Monitor Unit \\
MV & Mega Voltage \\
IMRT & Treatment Planning System \\
PDP & Standard Deviation \\
QA & Portal dose prediction \\
SDD & Quality Assurance \\
SAD & Source to Detector Distance \\
SD & Source Axis Distance \\
TPS & Stated Radiation Therapy \\
\hline &
\end{tabular}

